\title{
"I don't like Mondays"-day of the week of coronary heart disease deaths in Scotland: study of routinely collected data
}

\author{
Christine Evans, James Chalmers, Simon Capewell, Adam Redpath, Alan Finlayson, James Boyd, \\ Jill Pell, John McMurray, Kate Macintyre, Lesley Graham
}

Information and Statistics Division, National Health Service in Scotland, Trinity Park House, Edinburgh EH5 3SQ

Christine Evans specialist registrar in public health medicine James Chalmers consultant in public health medicine Adam Redpath principal statistician Alan Finlayson senior health information scientist James Boyd senior health information scientist Lesley Graham senior clinical medical officer

Department of Public Health, University of Glasgow, Glasgow G12 8RZ

Simon Capewell senior lecturer

Kate Macintyre medical epidemiologist

Department of Cardiology, University of Glasgow, Glasgow Royal Infirmary, Jill Pell homorary clinical senior lecture continued over Glasgow G31 2ER

A recent study in Moscow reported higher cardiovascular mortality on Saturdays, Sundays, and Mondays and linked this to the Russian pattern of binge drinking. ${ }^{1}$ Other studies have reported an increased risk of cardiac events on Mondays, a peak on other days, or no significant weekly variation. ${ }^{2}$ We investigated the patterns of death from coronary heart disease by day of the week in the Scottish population and explored possible links with patterns of alcohol consumption.

\section{Subjects, methods, and results}

Data linking all hospital discharges and deaths were obtained from the Scottish Morbidity Record and General Register Office (Scotland) respectively for all Scottish residents dying in 1986-95 for whom coronary heart disease (ICD-9 (international classification of diseases, 9th revision) codes 410-414) was the principal cause of death. Information was obtained on whether death occurred in hospital and whether subjects had been admitted to hospital with coronary heart disease since 1981. People dying in Scotland from all other causes in 1986-95 formed a comparison group. The $\chi^{2}$ test was used to examine differences between the observed number of deaths per day and that expected in the absence of any weekly variation. Emergency admissions to hospital in Scotland for non-dependent alcohol abuse (ICD-9 code 305.0) were used as a proxy for excess alcohol consumption.

During 1986-95, 91193 men and 79051 women died from coronary heart disease. Overall there was a significant weekly variation $(\mathrm{P}<0.001)$, with an excess of deaths on Monday ( $3.1 \%$ above the daily average). Subgroup analysis showed that this reflected a

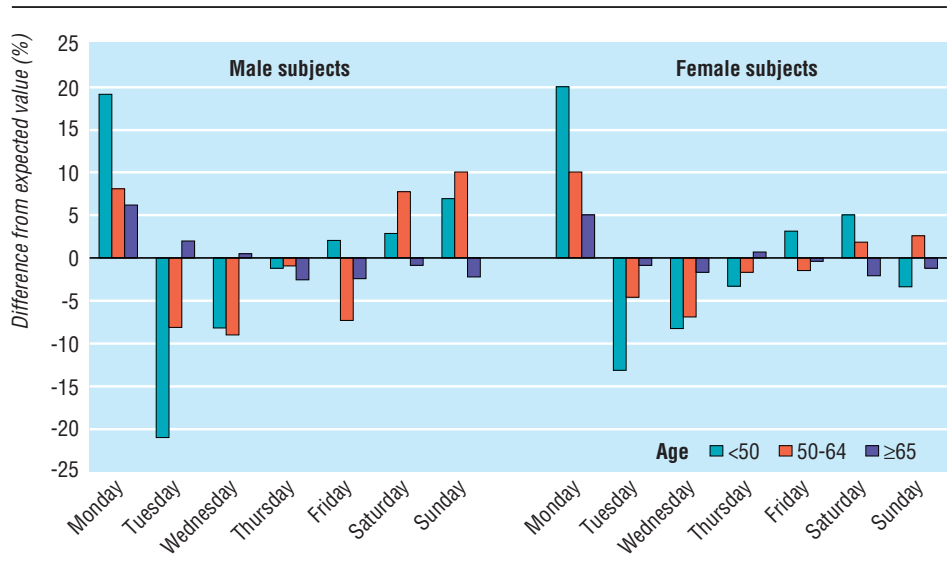

Deaths from coronary heart disease occurring outside hospital, by day of week, in people with no previous admissions for coronary heart disease, Scotland, 1986-95: percentage difference from expected values significant weekly variation among the 58448 people with no previous admission for coronary heart disease dying outside hospital (figure). Within this group, the Monday excess was greatest in those aged under 50 (men $19.2 \%$ above the daily average $(\mathrm{P}<0.01)$; women $20.0 \%$ above the daily average $(\mathrm{P}=\mathrm{NS}))$. Men under 65 also showed a highly significant excess of deaths on Saturday $(\mathrm{P}<0.01)$ and Sunday $(\mathrm{P}<0.001)$.

There was no significant weekly variation among people with a previous admission for coronary heart disease dying from coronary heart disease outside hospital $(n=18574)$ and no consistent weekly trend among patients dying from coronary heart disease in hospital ( $n=93$ 222). Deaths from all other causes $(\mathrm{n}=449345)$ exceeded expected values on Thursday and Friday and peaked on Saturday at $1.1 \%$ above the daily average $(\mathrm{P}<0.01)$. There was a highly significant weekly variation in emergency admissions for nondependent alcohol abuse $(\mathrm{P}<0.001)$, consistent with a pattern of binge drinking on Friday, Saturday, and Sunday $(20 \%, 64 \%$, and $11 \%$ above the daily average respectively).

\section{Comment}

We found an excess of deaths from coronary heart disease outside hospital on Mondays among people with no previous admission for coronary heart disease. This excess was different from the weekly pattern in deaths from all causes. It was unlikely to be an artefact of deaths remaining undiscovered until Monday because this pattern was not seen in people with a previous admission for coronary heart disease dying outside hospital.

The Monday peak in deaths from coronary heart disease in Scotland may be partly attributable to increased drinking at the weekend, although other mechanisms, such as work related stress, may be important. ${ }^{2}$ Several potential mechanisms have been suggested linking cardiovascular disease and death with binge drinking and alcohol withdrawal. ${ }^{3}$ We observed no Monday excess in people with a previous admission for coronary heart disease dying of coronary heart disease outside hospital. This group may be partly protected from sudden cardiac death by current treatment ${ }^{4}$ or may be more likely to seek medical help at the weekend because of familiarity with the symptoms. The possible link between binge drinking and deaths from coronary heart disease has potentially important public health implications and merits further investigation.

Contributors: CE contributed to the initial design, performed the analyses and interpretation, and wrote the first draft. JC had the original idea for the study, contributed to the design and 
interpretation, and wrote the final version. SC contributed to the initial design, interpretation, and writing of the paper. $\mathrm{AF}$, $\mathrm{JB}$, and AR performed the data extractions and analyses and contributed to the interpretation and drafting. JP, JMcM, and $\mathrm{KM}$ contributed to the interpretation and drafting. LG contributed to the extraction and interpretation of data relating to alcohol consumption and to drafting. SC will act as guarantor.

Funding: The study was funded by the British Heart Foundation.

Competing interests: None declared.
1 Chenet L, McKee M, Leon D, Shkolnikov V, Vassin S. Alcohol and cardiovascular mortality in Moscow; new evidence of a causal association. J Epidemiol Community Health 1998;52:772-4.

2 Willich SN, Lowel H, Lewis M, Hormann A, Arntz H-R, Keil U. Weekly variation of acute myocardial infarction. Increased Monday risk in the working population. Circulation 1994:90:87-93.

3 McKee M, Britton A. The positive relationship between alcohol and heart disease in eastern Europe: potential physiological mechanisms. J R So Med 1998;91:402-7

4 Peters RW. Circadian patterns and triggers of sudden cardiac death. Cardiol Clin 1996;14:185-94.

(Accepted 6 October)
Department of Cardiology,

University of

Glasgow, Western

Infirmary, Glasgow G12 8QQ

John McMurray

professor

Correspondence to: J Chalmers Jim.Chalmers@isd. csa.scot.nhs.uk

\section{Drug points}

\section{Systemic granulomatous disease after intravesical BCG instillation}

F C Mooren, M M Lerch, H Ullerich, H Bürger, W Domschke Departments of Medicine B and Pathology, Westfälische Wilhelms-Universität, Münster, Germany

Vaccination with BCG, an attenuated strain of bovine tubercle bacilli, has been used for most of this century to protect against tuberculosis. ${ }^{1}$ Recently the cell mediated immune response that follows inoculation with this organism was found to be an effective treatment for different types of cancer. ${ }^{2}$ We report a patient in whom application of BCG into the urinary bladder was quite effective in controlling a superficial vesical carcinoma but who developed granulomatous disease of multiple organ systems.

A 75 year old man was admitted because of a two month history of recurrent fever up to $40^{\circ} \mathrm{C}$. His history was unremarkable except that eight months earlier a transitional cell carcinoma of the urinary bladder (pT1) had been endoscopically resected and treatment with monthly intravesical instillations of BCG was begun. ${ }^{3}$ The last instillation had been given one month before the present admission. Laboratory studies on admission revealed thrombocytopenia $\left(82 \times 10^{9} / 1\right)$ and abnormal levels of aspartate aminotransferase (40 $\mathrm{U} / \mathrm{l}$, normal $<18)$, $\gamma$-glutamyltransferase (254 U/1, normal <28), alkaline phosphatase (566 U/l, normal <180), cholinesterase (1534 U/1, normal >3000), and pancreatic lipase (720 $\mathrm{U} /$, normal < 190). Computed tomography of the chest revealed a "ground glass" infiltration and micronodular pattern near the pleural space, and both a liver biopsy and a bone marrow sample (figure) revealed numerous non-caseating granulomas with epitheloid and giant cells An attempt to cultivate mycobacteria from these specimens was unsuccessful. Although repeated cultures of blood, sputum, and gastric juice were also negative, Mycobacterium bovis was found in urine samples. After treatment with isoniazid, rifampicin, and ethambutol, clinical recovery was prompt: the fever quickly resolved, and within a week laboratory studies showed normal values. When tuberculostatic treatment was discontinued five months later no relapse occurred.

Since its development in 1910, more than 1.5 billion people have received BCG vaccine as a prophylaxis against tuberculosis, and its administration is generally considered safe. ${ }^{3}$ More recently BCG instillation into the bladder has become an established and highly effective treatment for in situ and recurrent low grade bladder cancer. ${ }^{2}$ Although complications from intravesical appli-

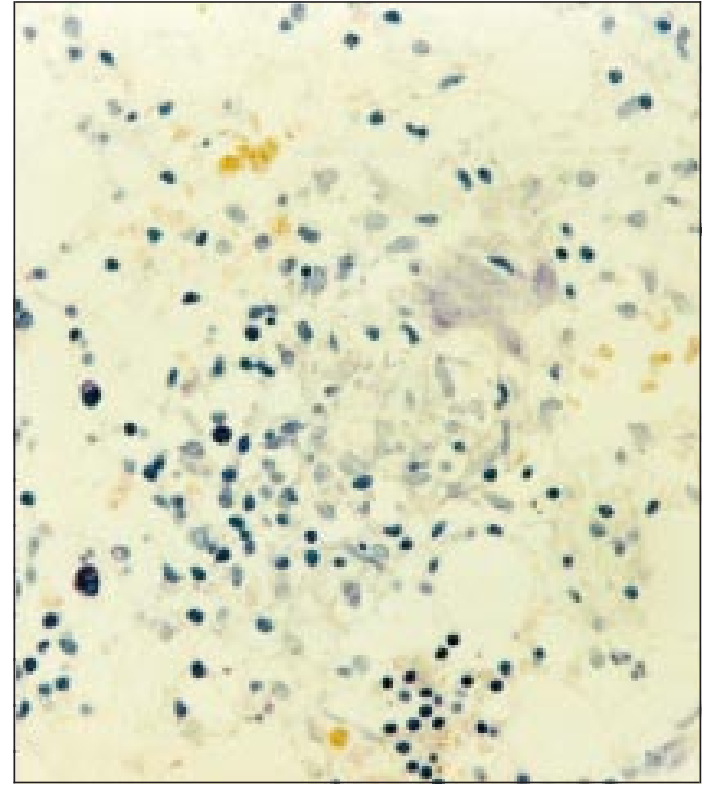

Sarcoid-like granuloma in an otherwise normal bone marrow sample

cation of BCG have occasionally been observed, ${ }^{4}$ such extensive systemic complications as we describe have not previously been reported. This patient's clinical course suggests that systemic granulomatous disease, and bone marrow involvement in particular, should be considered when a patient develops symptoms of systemic infection after inoculation with BCG. Given the extended period during which BCG can be excreted, patients who undergo this treatment should also be advised to disinfect their urine to avoid infecting others.

1 Zumla A, Grange J. Clinical review-tuberculosis. BMJ 1998:316:1962-4

2 Lamm DL, Blumenstein BA, Crawford ED, Montie JE, Scardino P, Grossman $\mathrm{HB}$, et al. A randomized trial of intravesical doxorubicin and immunotherapy with bacille Calmette-Guerin for transitional-cell carcinoma of the bladder. N Enol J Med 1991:325:1205-9.

3 Lotte A, Wasz-Hockert O, Poisson V, Dumitrescu N, Verron M, Couvet E BCG complications. Estimates of the risks among vaccinated subjects and statistical analysis of their main characteristics. Adv Tuberc Res 1984;21:107-93

4 Lamm DL, van der Meijden PM, Morales A, Brosman SA, Catalona WJ, Herr HW, et al. Incidence and treatment of complications of bacillus Calmette-Guerin intravesical therapy in superficial bladder cancer. J Urol 1992;147:596-600. 